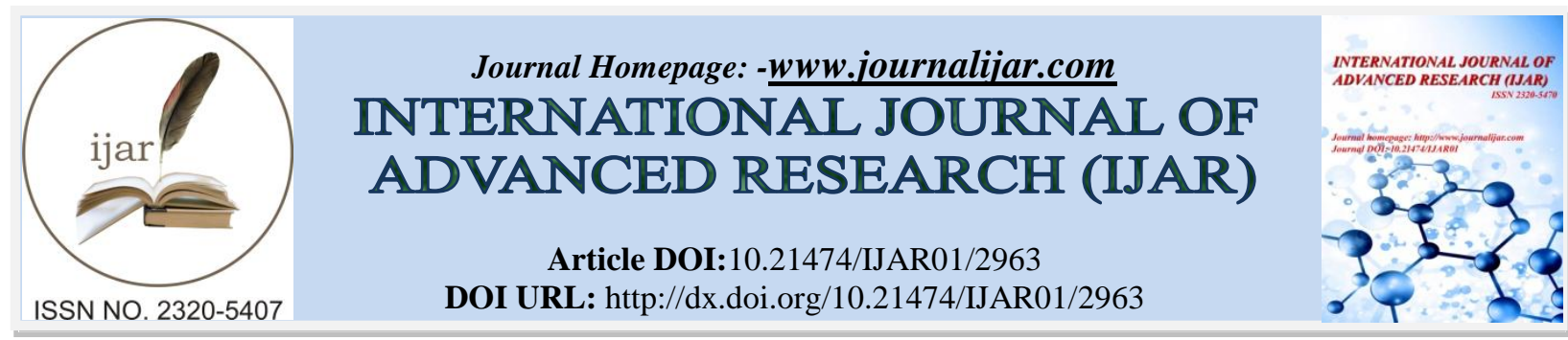

RESEARCH ARTICLE

\title{
THE TRENDS OF GALLSTONE SURGICAL TREATMENT IN SAUDI ARABIAN PROVINCE: IS THE LOCAL PRACTICE UP TO DATE?
}

\author{
Anthony Morgan, Mohammed K Alelwan, Naif Alqahani, Ahmed Alqahtani, Humaidan Almalki, Abdullah \\ Alshaalan and Sultan Almutrafi.
}

\section{Manuscript Info}

Manuscript History

Received: 30 November 2016

Final Accepted: 26 December 2016

Published: January 2017

\begin{abstract}
Gallstone disease continues to be one of the leading problems in modern gastroenterology and in majority of cases requiring surgical management. This pathology has large impact on Saudi Arabian population and its Health system. Laparoscopic cholecystectomy has become a procedure of choice in current management of acute and chronic cholecystitis. The aim of this surgical audit based study was to analyze current management outcome in selected group of patients, who underwent laparoscopic cholecystectomy procedure in Saudi Arabian province of Al Kharj with relatively large local population. The multiple factors including patient's demographic characteristics, modality and results of investigations, as well as, surgical outcome were analyzed and compared. This study demonstrated satisfactory surgical outcomes in gallstone management in the Prince Sattam Bin Abdulaziz University affiliated hospital, which demonstrated low complications rate and cost effectiveness.
\end{abstract}

Copy Right, IJAR, 2016,. All rights reserved.

\section{Introduction:-}

Gallstones are known to medical practice since ancient times and remain one of the most frequent pathological conditions of the gastrointestinal tract in modern time. The high prevalence gallstone diseases more shown in developed countries reaching from 10 to $20.8 \%$. However, it is less common in the developing nations and remaining as low as $4.1 \%$ (1). At the same time majority (about $80 \%$ ) of the patients with gallstones remain asymptomatic during their entire life, and only about $3 \%$ of patients with periodical symptoms may develop acute cholecystitis, which require eventual surgical intervention for removal of gall bladder.

It was estimated that about $20 \%$ of adults at the age of 40 and over would develop biliary calculi. This figure may be increased by $30 \%$ by the age of 70 and above. Female-to-male ratio is reaching about $4: 1$ in the reproductive years, and almost equal in the older population (2).

It appears that, factors like diet and socio-economic conditions are directly correlated with the incidence of gallstone disease. Risk factors known to contribute to the development of gall stones also include the family history of gallstone, obesity, multiple pregnancies, fat diet, and low physical activity lifestyle. Some co-morbidities, which have physiological predisposing factors like abnormal absorption in biliary digestive system, such as,Crohn's disease and liver related pathology also increase gall stone formation. 
Specific clinical features supported by ultrasound scanning evidence are base for establishing the diagnosis of acute cholecystitis and define the reasoning for surgical treatment. However, the timing of surgery still debatable, and up to date there is no standard approach among different surgical schools.

The aim of this study was to investigate current trends in approach of investigation and management of gallstone disease patients from the population area in search of the possibility of any improvement in outcome and hospital stay.

\section{Materials and Methods:-}

This retrospective surgical audit study was based on the identified hospital database, obtained from medical records at King Khaled Hospital $(\mathrm{KKH})$, which is a teaching hospital affiliated with the Prince Sattam Bin Abdulaziz University in Al KharjGovernate of Saudi Arabia covering the population of over 650,000 people.

Medical records of 132 patients admitted from 2010 to 2015 inclusive, who presented with acute or chronic cholecystitis and received surgical treatment as emergency or elective cases, were reviewed.

Inclusion criteria for this study were all patients, who had laparoscopic or open cholecystectomies, presenting to KKH with the diagnosis of acute or chronic cholecystitis or symptomatic cholecystolithiasis.

Patients were discriminated by the gender and included in two major groups, such as the emergency case or elective case. Patient's characteristics were divided in few sections: 1) symptoms upon the presentation and family history, 2) blood inflammatory and biochemical values, such as white cell count (WCC) and Liver Function Test (LFT), 3) evaluation of gall stones together with common bile duct measurement by ultra-sonography (US) 4) types of surgical procedures and related complications, and 5) duration of hospital stay.

All patients had ultrasonography study to confirm the presents of gallstones and evaluate the severity of acute cholecystitis as well as exclude the gallstones in the common bile duct, which may change the strategy for the patient's management.

All of the patients received prophylactic antibiotics before the surgery or intra-operatively in order to prevent the possibility of infection.

Data for all these characteristics obtained from the medical records of the patients was analyzed for statistical value.For analysis of data the SPSS version 23 (Statistical Package for the Social Sciences) and Excel for Mac 2016 computer software programs were used in this study, which included unpaired $t$-test for comparison of means within groups, where $\mathrm{p}<0.05$ was considered as statistically significant.

\section{Results:-}

In total, 132 patient's data was collected from medical records and analysed, including 122 females and 10 females (92.4\% and $7.6 \%$ respectively). For the majority of the patient's age distribution falls in the 4th decade. The median age for males was 41.1 year (range 26-68 year) and for females 39.5 years (range 20-75 years) respectively. Distribution of age, gender and type of surgical intervention is shown in the Graph 1. 
Graph 1:-Distribution of age, gender and type of surgical intervention.

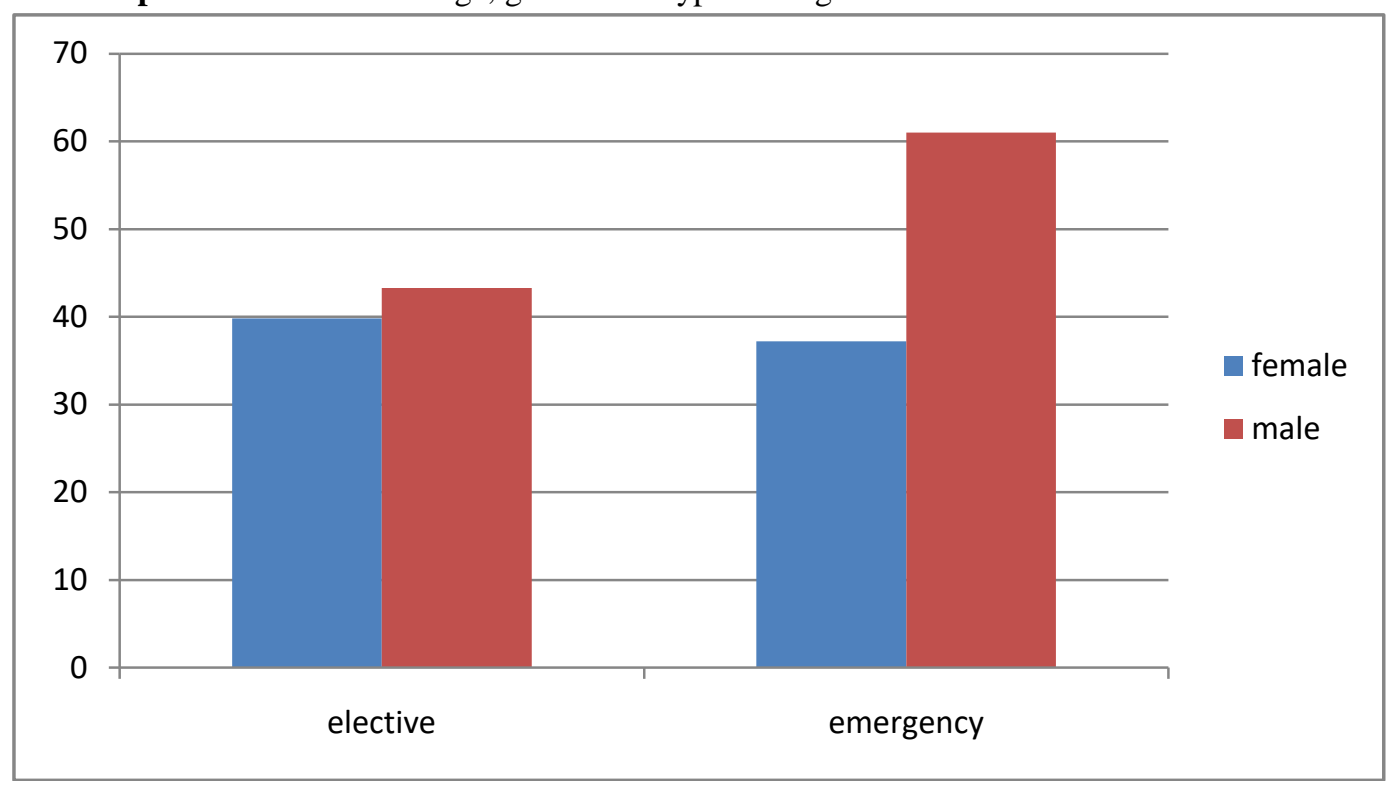

The median age in the elective surgery group was with male to female ratio 1:11.9. This was not much statistically different compare to the emergency group where median age was and male to female ratio $1: 15(\mathrm{p}>0.8)$. In both groups the diagnosis of cholecystitis was made on the basis of clinical presentation, evaluation of inflammatory markers, liver function tests (LFTs) results and ultrasound evidence of gallstones in all patients admitted for treatment.

Regarding the symptoms on the presentation to the hospital, nausea with vomiting were experienced by $67.2 \%$ of the patients in elective group, and $87.5 \%$ in emergency group respectively. Intermittent or constant right upper abdominal pain was present in $98.2 \%$ of patients in selective group and $93.8 \%$ in emergency group, which made this symptom as most constant. The characteristics of the symptoms in elective and emergency cases shown in the Table 1.

Table 1:- Characteristics of the presenting symptoms.

\begin{tabular}{|c|c|c|}
\hline Symptoms on admission & $\begin{array}{c}\text { Elective } \\
(116)\end{array}$ & $\begin{array}{c}\text { Emergency } \\
(16)\end{array}$ \\
\hline Nausea and vomiting & $78(66.7 \%)$ & $12(70.6 \%)$ \\
\hline Right upper abdominal pain & $114(97.4 \%)$ & $15(88.2 \%)$ \\
\hline Epigastric pain & $80(68.4 \%)$ & $15(88.2 \%)$ \\
\hline Pain in tip of the right shoulder & $12(10.3 \%)$ & $4(23.5 \%)$ \\
\hline
\end{tabular}

There was no jaundice in elective group, however, 3 out 16 patients (18.8\%) in emergency group presented with jaundice. None of the elective patients had concurrent pancreatitis compare to emergency group, where pancreatitis was evidently diagnosed in 37,5\% of patients. Only one patient in elective group was with chronic liver disease, however, no chronic liver pathology was identified in the emergency group of patients.

Elevated bilirubin with the deranged liver function test was found in 11 patients, where 10 patients belonged to emergency group. Mean values for levels of bilirubin and liver function test in elective and emergency groups shown in the Table 2. 
Table 2:-Mean values for levels of bilirubin and liver function test.

\begin{tabular}{|c|c|c|c|c|}
\hline & \multicolumn{2}{|c|}{$\begin{array}{c}\text { Elective } \\
\text { Emergency } \\
\text { (mean +/- SD) }\end{array}$} & \multicolumn{2}{c|}{ +/- SD) } \\
\hline Bilirubin (umol/L) & 20.06 & 11.9 & 21.9 & 14.5 \\
\hline ALT (u/L) & 78.8 & 48.7 & 575.4 & 141.6 \\
\hline AST (u/L) & 44.2 & 29.2 & 384.4 & 141.6 \\
\hline ALP $(u / L)$ & 89.7 & 48.4 & 184.8 & 54.0 \\
\hline
\end{tabular}

Biliary dilatation on ultrasound study was found in 17 out of 116 elective patients (14.7\%), while in emergency group it was reaching $43.7 \%$ respectively.

Gallstones on ultrasound were diagnosed in 115 out of 116 patients in elective group and in 15 out of 16 in the emergency group respectively. Biliary dilatation identified by ultrasonography study was found in $17.2 \%$ of patients in elective group compare to $77.7 \%$ in emergency group.

In 4 out of 116 patients (3.4\%) laparoscopic cholecystectomy had to be converted to open procedure due to technical difficulties in dissecting Calot's triangle in order to identify important biliary structures, which could be challenging because of the extensive scarring process after gall bladder inflammation. There was no conversion to open procedure in the emergency group of patients, as acutely inflamed tissues helping in laparoscopic dissection, but increasing the chance of intra-operative bleeding.

In our study, median length of hospital stay (LHS) was 2.7 days ranging from 1 to 18 days. For the elective group the median LHS was 2.5 days, while in the emergency group it was 4.7 days respectively (p<0.03) In total 3 patients $(2.3 \%)$ had complications, 1 intra-operative bleeding in the elective group and 2 postoperative wound infections, when the laparoscopic procedure had to be converted to open.

The diagnosis of chronic cholecystitis was associated with gallstones in 115 out of 116 elective surgery patients (99\%) and acute cholecystitis in all 16 patients from emergency group.

There was no intra or post-operative mortality in this study. Intra-operative perforation of the gall bladder and bleeding were considered as well recognized procedural complications. Only one intra-operative bleeding has occurred in the whole series of cholecystectomies for elective group and no bleeding was recorded in emergency group. Post-operative wound infection has developed in one patient from each group during post-operative period.

\section{Discussion:-}

The prevalence and incidence of gallstones in Saudi Arabia is not well established, although recent studies demonstrate high numbers, which vary from region to region (3). Abu-Eshy et al (2007) in their cross-sectional community-based study of gallstone disease in a Saudi Arabian Asir region demonstrated that the overall prevalence was $11.7 \%$. This study was based on abdominal ultra-sonography with structured interview and shown that hypochondrium pain was significantly correlated with gallstone disease (4).

The trends for changes in diet habits in Saudi Arabia demonstrated the increase of fat and sugar consumption with high calories intake and reduction of high-fiber food, which is related to introduction of the fast food and more sedentary life style among the younger generation over the past few decades. This leads to the higher number of population with increased body mass index (BMI) among Saudis. Also, international reports shown that female population more effected by the early formation of gall stone and development of cholecystitis compare to male population. Multiple pregnancies, which are traditional for Saudi females appeared as one of the risk factors for cholelithiasis in this population group. The formation of cholesterol sediments, so called"gall bladder sand" occurs more frequently compare to males. In our study $28.2 \%$ of females had multiple pregnancies.

Malatani et al. (1996) in their study demonstrated that, cholesterol was the most common component of the gallstones (82.6\% to 90.4\%) in various regions of Saudi Arabia, which was followed by calcium, bilirubin, and carbonate (5). 
The presents of gallstone disease may have no specific symptoms, as the majority of the population with gallstones (70-90\%) can remain asymptomatic until complications develop, which include acute cholecystitis, cholangitis or biliary pancreatitis. In our study, dyspeptic symptoms were more prominent in the emergency group of patients compare to the elective $(70.6 \%$ vs $66.7 \%$ respectively) $(\mathrm{p}<0.05)$, which correlates well with more acute condition supporting the diagnosis of severe cholecystitis or development of pancreatitis and influencing the decision making to perform cholecystectomy as emergency.

Diagnosis of acute or chronic cholecystitis was made based on symptoms and signs of localized inflammation in epigastric and the right upper quadrant areas and differentiated from biliary colic by the presence of elevated inflammatory markers and liver function test. Patients may present with a history of biliary colic or may have been previously asymptomatic.

Plain X ray films have never been a reliable diagnostic tool in detecting gallstones, as the frequency of radio-opaque stones is low and can be demonstrated only in $10-15 \%$ of cases.

Ultrasonography currently is gold standard non-invasive method for clinical diagnosis of gallstones and cholecystitis with sensitivity reaching $98 \%$. However, it can be inconclusive in some cases as remain operator sensitive. Its ability to demonstrate the presence ofpericholecystic fluid, gall bladder distention with the wall thickness and gallstones with their location and common bile duct dilatation make it the method of choice in diagnosis and screening. Together with color Doppler it can show local hyperemia and severity of the inflammation.

Biliary dilatation in the elective group (17.7\%) could be explained as a transient passage of the gallstone into duodenum, as there was no record of pancreatitis in this group. However, in the emergency group, biliary dilatation was $77.7 \%$, which correlates well with development of pancreatitis in $60 \%$ of emergency patients $(\mathrm{p}<0.05)$.

Today, laparoscopic cholecystectomy is a gold standard procedure in the management of gallstones. The technological advances and evolution of laparoscopic technique require continues reeducation and retraining by surgeons.

About $20 \%$ of patients with acute gall bladder inflammation would require emergency surgery with deterioration of the patient's condition and formation of localized peritonitis due to empyema of gall bladder.

However, the optimal timing of surgery for the rest $80 \%$ of patients continues to be debatable. Multiple studies suggest that early surgery has reduced complications and lower conversion rate as well as shorter hospital stay. Early laparoscopic surgery, less than 72 hours after presenting symptoms, allow easier dissection of edematous planes before the fibrous scarring process occur (6).

In the large randomized trial by Gutt et al. (2013), laparoscopic cholecystectomy, performed within first 24 hours of hospital admission demonstrated superior results compare to conservative approach regarding morbidity and cost effectiveness (7).

In our study intraoperativecholangiogram (IOC) was used selectively only in cases of high suspicion of gallstones in common bile duct (CBD), equivocal ultrasound findings or transient pancreatitis.

Polat et al. (2000) documented findings of CBD stones in up to $12 \%$ of all patients during cholecystectomy and claim that intra-operative cholangiography (IOC) is effective in identifying stones in CBD during surgery.Thus, theyrecommend routine IOC to identify duct anatomy in order to reduce the incidence of CBD injury (8). This recommendation supported by the Ragulin-Coyne et al (2013), who reached similar conclusions (9). Moreover, gallstone complications, such as biliary pancreatitis and CBD injury during laparoscopic cholecystectomy can dramatically increase the cost of recurrent hospitalization by 10 fold, as well as morbidity and mortality of patients with the gallstone pathology.

\section{Conclusion:-}

Laparoscopic cholecystectomy is an operation of choice in the management of gallstones and in case of acute cholecystitis should be used earlier after the onset of symptoms. In many cases it is important action to prevent the variety of complications caused by gallstones. 
Intra-operative cholangiogram is valuable methods in depicting biliary anatomy, which may exclude bile duct injuries and identify stones in common bile duct. Thus, indicating to further necessity for bile duct exploration or to perform ERCP modality to prevent gallstone pancreatitis.

Our study demonstrate that current management of acute and chronic cholecystitis in Al KharjGovernate area has relatively low rate of complications and sufficiently cost effective. This could be explained by careful and appropriate diagnosis and timing for surgery in majority of the cases, included in this study.

\section{References:-}

1. Jarrar B.M., MeshrefA Al-Rowaili M.A.,Chemical Composition of Gallstones from Al-Jouf Province of Saudi Arabia, Malays J Med Sci. 2011 Apr-Jun;18(2):47-52.

2. Schirmer BD, Winters K., Edlich R. Cholelithiasis and CholecystitisJLong Term Eff Med Implants 2005, v15,:329-338.

3. Tamimi TM, Wosomu L, Al Khozaim A, Abdul-Ghani A. Increased cholecystectomy rates in Saudi Arabia. Lancet 1990;336:1235-7.

4. Abu-Eshy SA, et al. Prevalence and risk factors of gallstone disease in a high altitude Saudi population. East Mediterr Health J. 2007 Jul-Aug; 13(4):794-802.

5. Malatani TS, Bobo RA, Al-Kassab AS, et al. Gallbladder stones analyzes, bile and wound cultures in cholelithiasis. Saudi J Gastroenterol,1996;2:146-9.

6. Pessaux P, Tuech JJ, Rouge C, et al. Laparoscopic cholecystectomy in acute cholecystitis. A prospective comparative study in patients with acute vs. chronic cholecystitis. SurgEndosc. 2000;14:358-361.

7. Gutt $\mathrm{CN}^{1}$, Encke J, Köninger Jet al.,Acute Cholecystitis-early laparoscopic surgery versus antibiotic therapy and Delayed elective Cholecystectomy, Ann Surg. 2013 Sep;258(3):385-93.

8. Polat FR, Abci I, et al. The Importance of IntraoperativeCholangiography during Laparoscopic Cholecystectomy. JSLS, 2000 Apr-Jun; 4(2): 103-107.

9. Ragulin-Coyne E, Witkowski ER, Chau Z, et al. Is routine intraoperativecholangiogram necessary in the twenty-first century? A national view. J Gastrointest Surg. 2013 Mar; 17(3): 434-44. 